\title{
Numerical Analysis of Solar Fluxes Reflected by Hexagonal Shape Reflector Combination
}

\author{
Chang-Woo Son, Sang-Hun Lee, Ki-Hwan Song, Sang-Eun You, Woo-Hyeong Heo, Ki-Won Kim, \\ Jeong-Heon Lee, Kyum Kim, Wan-Taek Lee, Hyeon-Gu Kim, Jin-Seok Cho, and Tae-Il Seo*
}

\begin{abstract}
This paper presents an analysis of concentrated fluxes by solar collector system. This solar collector was combined by the same shape independent reflectors. Before fabricating the system, we carried out design process to gather all focuses of independent reflectors onto one point that would be placed on absorber. For this, TracePro was used to analyze reflected fluxes, and to determine exact positions and orientations of all reflectors. For this simulation, reflection rate and reflected area were chosen as variables to know which variable is dominant. Through this study based on Finite Element Method by using TracePro, the relationship between fluxes and these variables could be figured out.
\end{abstract}

Keywords - Solar collector, TracePro, Flux, Reflector

\section{INTRODUCTION}

$\mathrm{R}$ ECENTLY, the new renewable energy has established dominance over nuclear energy [1]. However, as for Korea, the nuclear power still has taken a far larger part, as of 2012, compared to the new renewable one. However the necessity of new renewable energy is emphasized because many problem comes up about nuclear power such as nuclear power plant explosion in Fukushima, Japan. Cost of solar power generation is fall to level of thermal power generation and it will continuously decrease. Thus, researches on solar power which is infinitely available and no pollution problem has become urgent request [2]-[4].

Concentrating solar collectors are used for mid-high temperature solar power system, and they are divided into Vacuum-Tube type, PTC (Parabolic Trough Concentrator) type, CPC (Compound Parabolic Concentrator) type, dish type, and Solar-Tower type in accordance with the geometry of the concentration form [5]-[6]. The dish of the solar system is used as power production or the Institute of Industrial Engineers staggered through a small development and many studies have been made in developed countries, including the United States. Currently, Korea Institute of Energy Research has developed, installed and managed plate-type solar collectors, and this institute has a significant technical skill [7]. However it has disadvantage of expensive production cost because this is not single module and the state of research about reflector's arrangement is desired. In this paper, single module independent reflectors were taken into account in order to develop light-weight solar collector system. The independent

Chang-Woo Son was with Incheon National University, Incheon, 22012 Korea.

Tae-Il Seo ${ }^{*}$ was with Incheon National University, Incheon, 22012 Korea. He is now with the Department of Mechanical Engineering, Incheon National University. reflector provides some advantages as follows: (1) the reflectors can be mass-producted by reflectively easy ways, (2) it is easy to increase or decrease amount of reflected area, and (3) it is easy to fabricate the whole system of solar collectors. Therefore, in this study our objective of this research is determining positions and orientations of all independent reflectors to concentrate all reflected fluxes on a focus point. For this, TracePro was used to analyze behavior of reflected fluxes by the independent reflectors.

\section{II.DESIGN OF INDEPENDENT REFLECTORS}

\section{A. Modeling of independent reflectors}

Firstly, hexagonal shape single module independent reflectors were taken into account because it is expected that they can effectively arranged to accomplish solar collector system. Side line length is $300 \mathrm{~mm}$ and the distance between reflectors is $50 \mathrm{~mm}$ by considering wind load. The position of absorber is placed on focus point and distance from center of solar collector system to focus point is $2,500 \mathrm{~mm}$. To easily determine positions and orientations of all reflectors, we grouped array-1, array- 2 and array- 3 by the order of arranged reflectors because they would be radial symmetrically placed. Fig. 1 shows grouped array-1, array-2 and array-3 that are radial symmetrically placed.

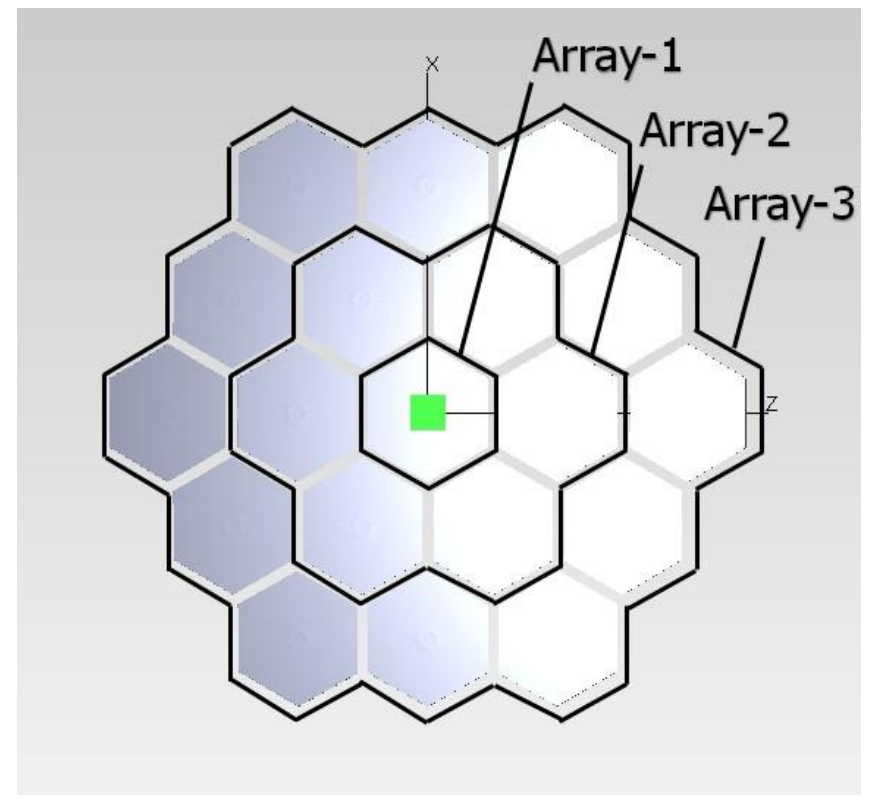

Fig. 1 Grouped array reflectors

NX 10 was used as modeling program. Array-1 was composed of only 1 reflector but array-2 was composed of 6 reflectors and array- 3 was composed of 12 reflectors. Each 
reflector area is $0.23 \mathrm{~m}^{2}$ and then total reflector area becomes $4.44 \mathrm{~m}^{2}$. All the reflector specification is the same and curved surface of designed reflector corresponds to aspheric surface to effectively collect solar flux.

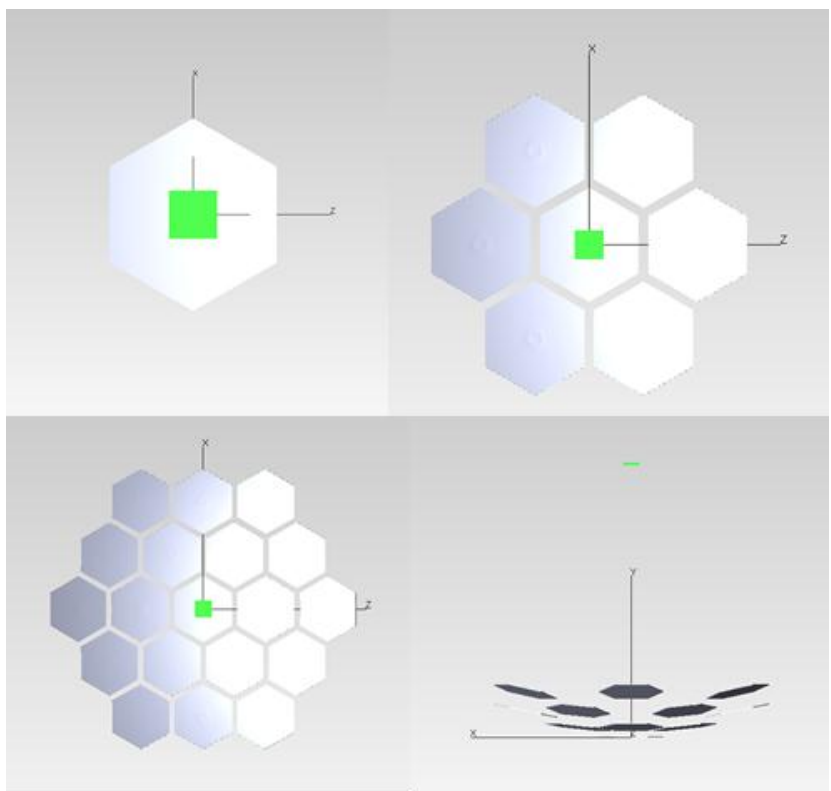

Fig. 2 Positions and orientations of arranged reflectors

Fig. 2 shows positions and orientations of arranged reflectors. It can be seen that each reflector was differently placed and oriented because all the reflectors must concentrate reflected solar flux onto only one focus point. As show in Fig. 1, all reflectors of array- 1 and array- 2 have the same positions and orientations because the distances from center of collector system to reflectors are the same. However the distances from the center to reflectors of array- 3 are different. Therefore from reflectors of array- 3 must be differently placed and oriented.

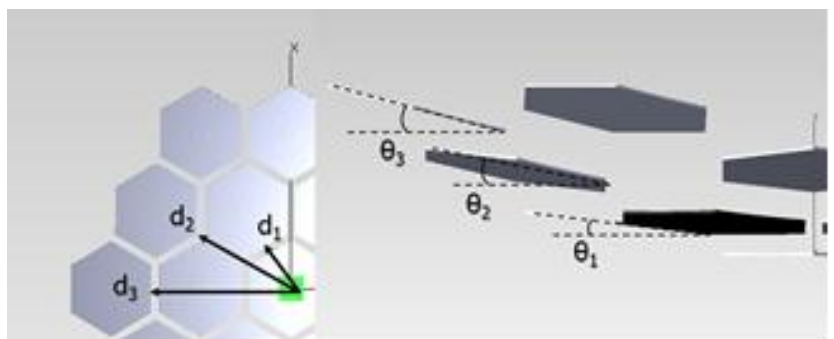

Fig. 3 Different positions and orientations of arranged reflectors

Fig. 3 shows different positions and orientations of arranged reflectors in 3-dimensional space. Fig. 4 shows four different points corresponding to angles, heights and distances of differently placed and oriented reflectors. This figure means that only four different positions and orientations of reflectors must be taken into account for designing three different arrays of reflectors. If more arrays have to be considered, it would be possible to consider only some typical positions and orientations of reflectors to design a solar collector system combined by the same independent reflectors. Fig. 5 shows a stirling engine that must be run by collected solar energy. The actual area of absorber is $150 \times 150 \mathrm{~mm}$. However $100 \times 100 \mathrm{~mm}$ rectangle was considered as the area of absorber in our numerical analysis to increase the accuracy of design.

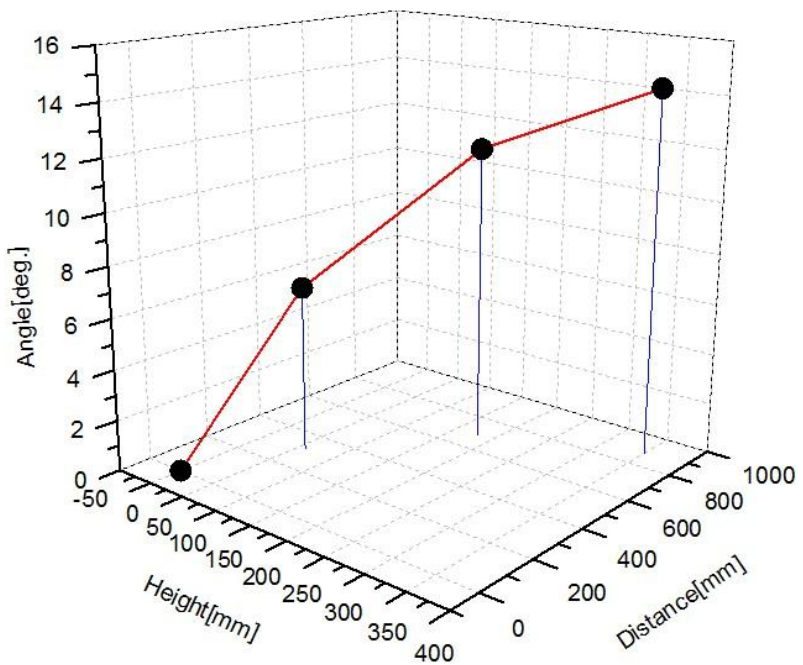

Fig. 4 Four different points corresponding to angles, heights and distances of differently placed and oriented reflectors

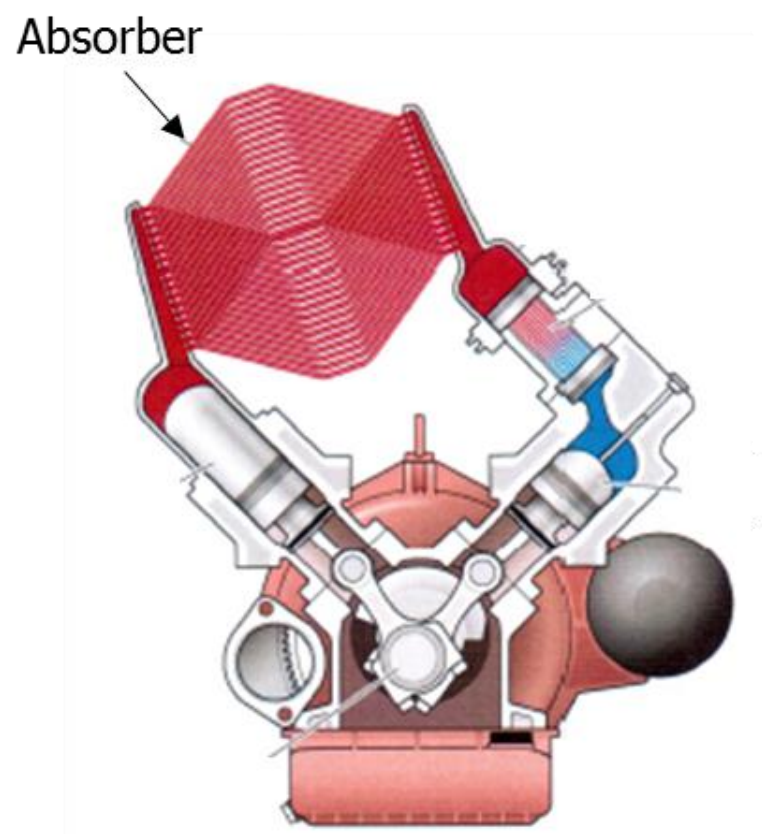

Fig. 5 Absorber of real stirling engine

\section{B. Boundary conditions for numerical analysis}

Fig. 7, 11, 15 shows simulation results of fluxes reflected by different array of reflectors and concentrated on the focus point by using TracePro. To determine positions and orientations of reflectors, TracePro was used by gradually controlling positions and orientations of reflectors until when concentrated focus can be placed on the center of absorber. Based on our past experimental research results, $800 \mathrm{~W} / \mathrm{m}^{2}$ of radiance was obtained when it was fine in summer time. Therefore this value was considered as average radiance just for simulation. Fig. 6 shows the radiance and temperature varied with time as our past experimental research results. It was assumed that the whole solar collector system vertically orient to solar rays. 85 , 90 and $95 \%$ of reflection rates were considered to carry out the simulation process. 


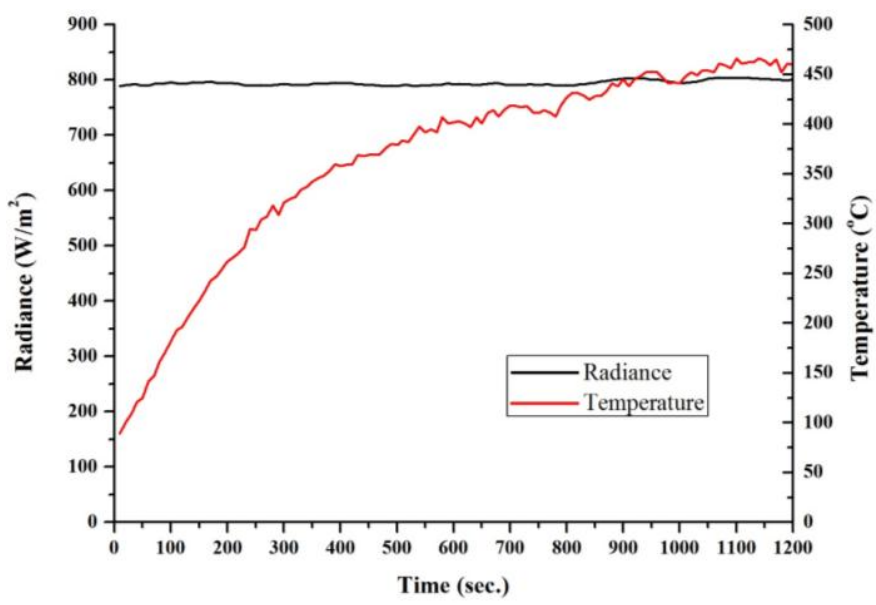

Fig. 6 Radiance and temperature varied with time

\section{SIMULATION RESULTS}

Fig. 7-18 show distributions of fluxes reflected by arranged reflector with 3 different reflection rates. Fig. 8-10 show distributions of fluxes reflected by array-1 reflector with 3 different reflection rates. Significant difference between 3 flux distributions cannot be seen in simulation results.

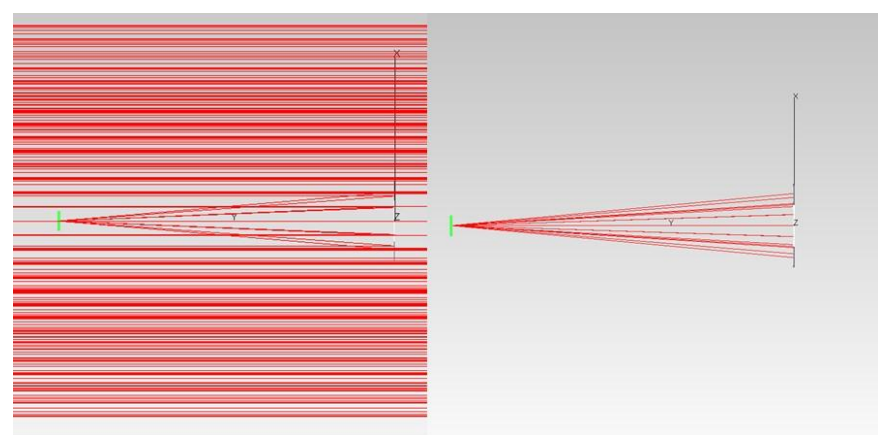

Fig. 7 Simulation of array-1 reflector

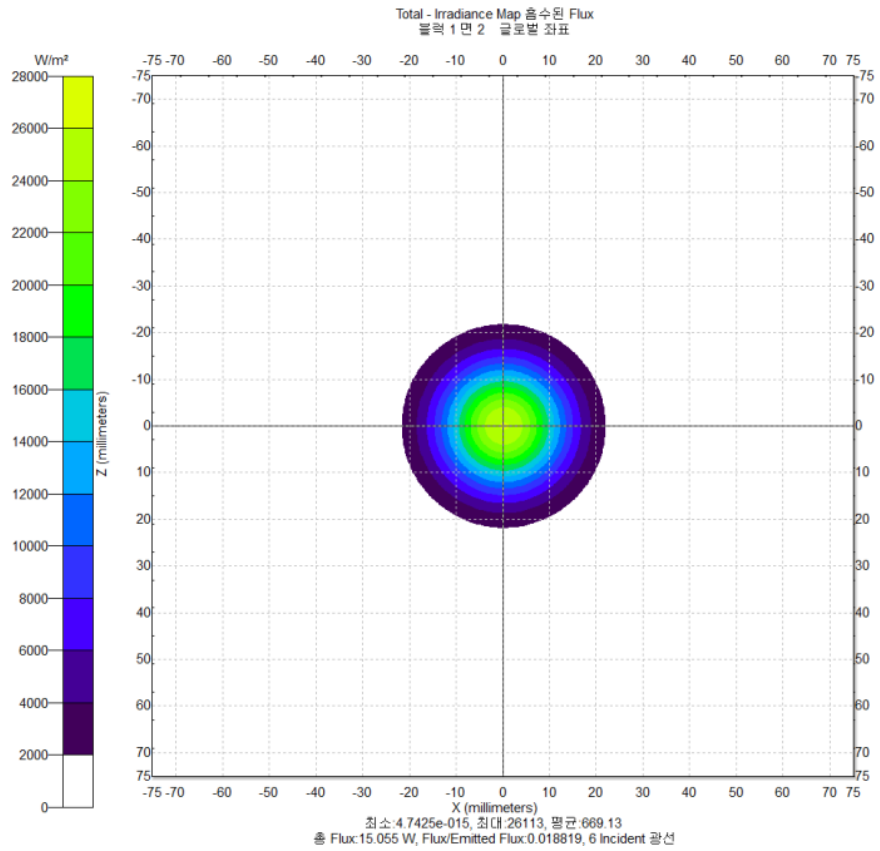

Fig. 8 Distribution of flux with $85 \%$ reflection rate of array-1
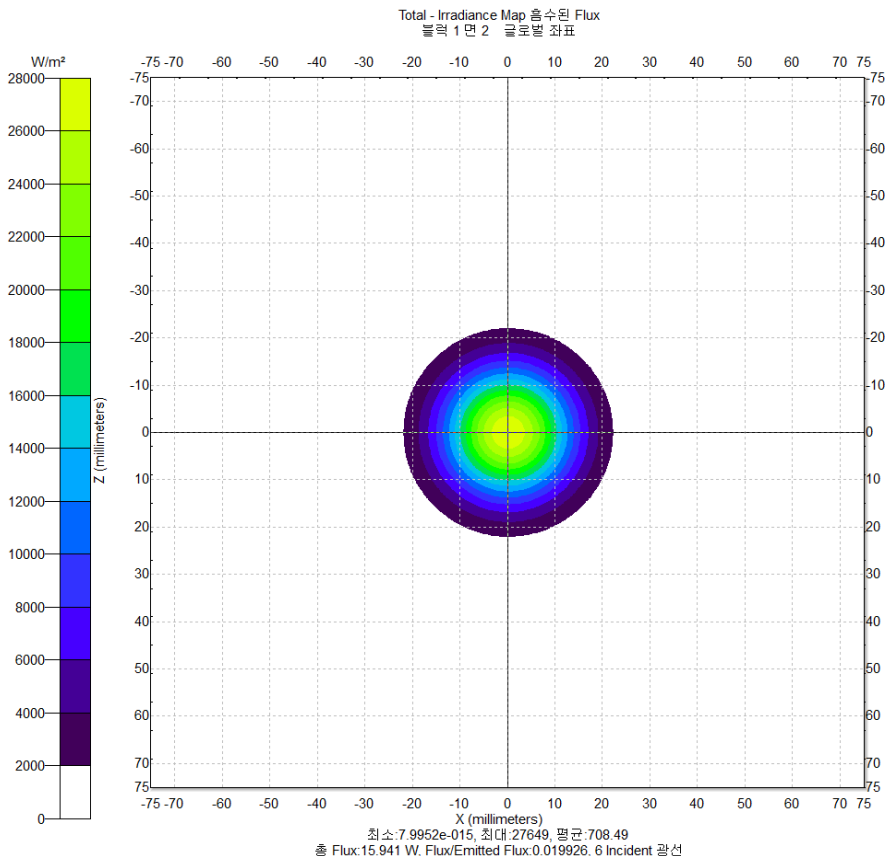

Fig. 9 Distribution of flux with $90 \%$ reflection rate of array-1
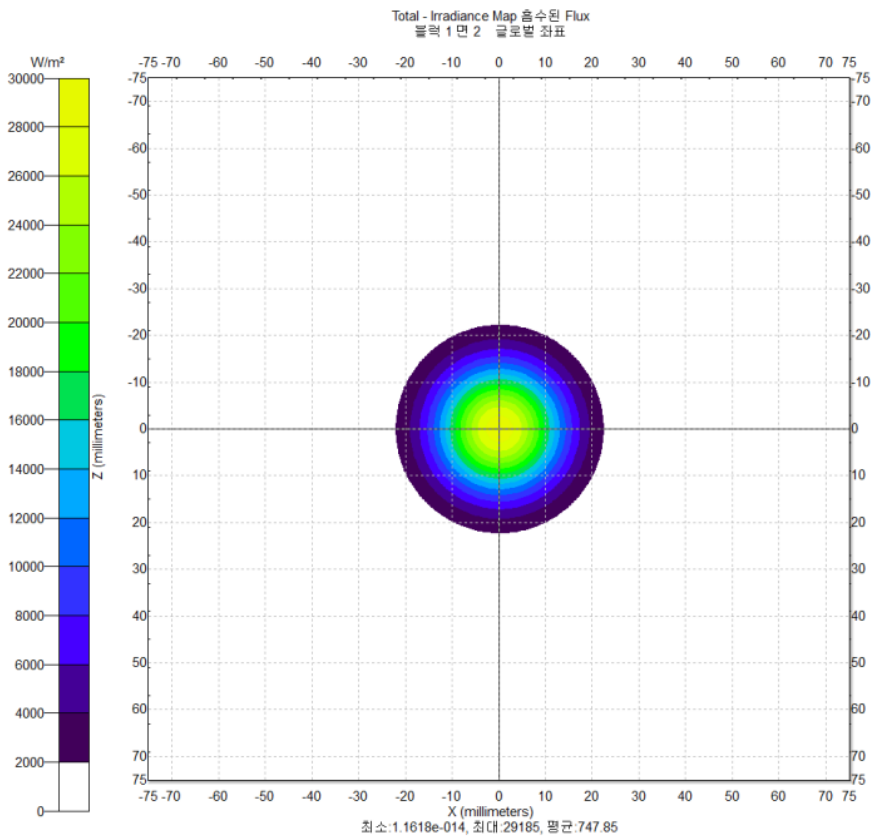

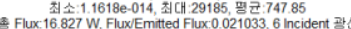

Fig. 10 Distribution of flux with 95\% reflection rate of array-1

Fig. 12-14 show distributions of fluxes reflected by array-2 reflector with 3 different reflection rates. Significant difference between 3 flux distributions cannot be also seen in simulation results.

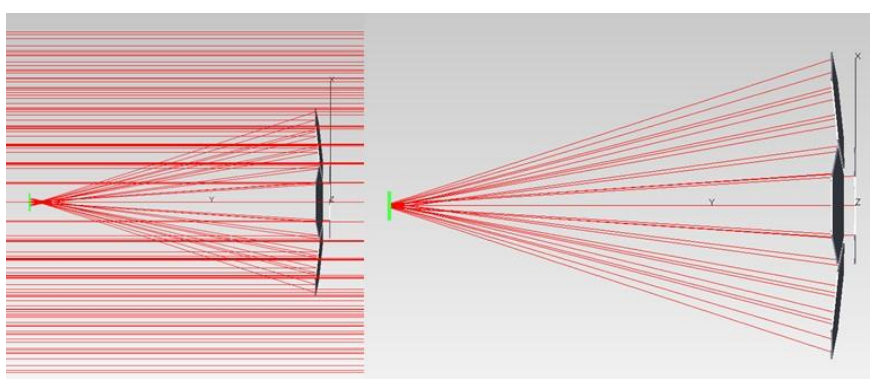

Fig. 11 Simulation of array-2 reflectors 


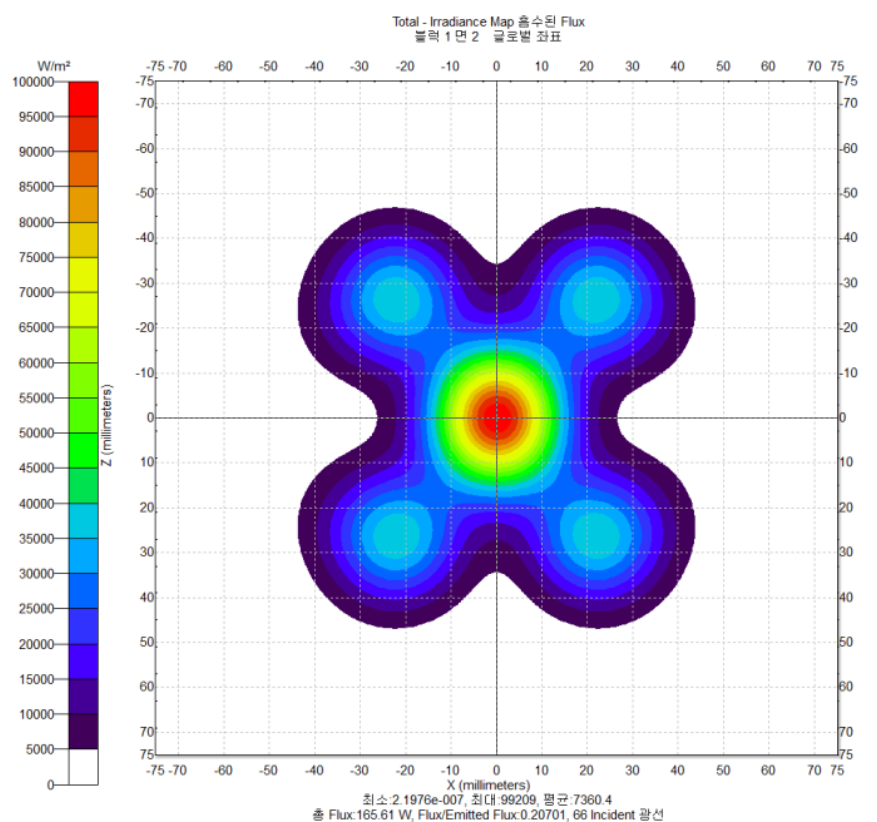

Fig. 12 Distribution of flux with $85 \%$ reflection rate of array-2

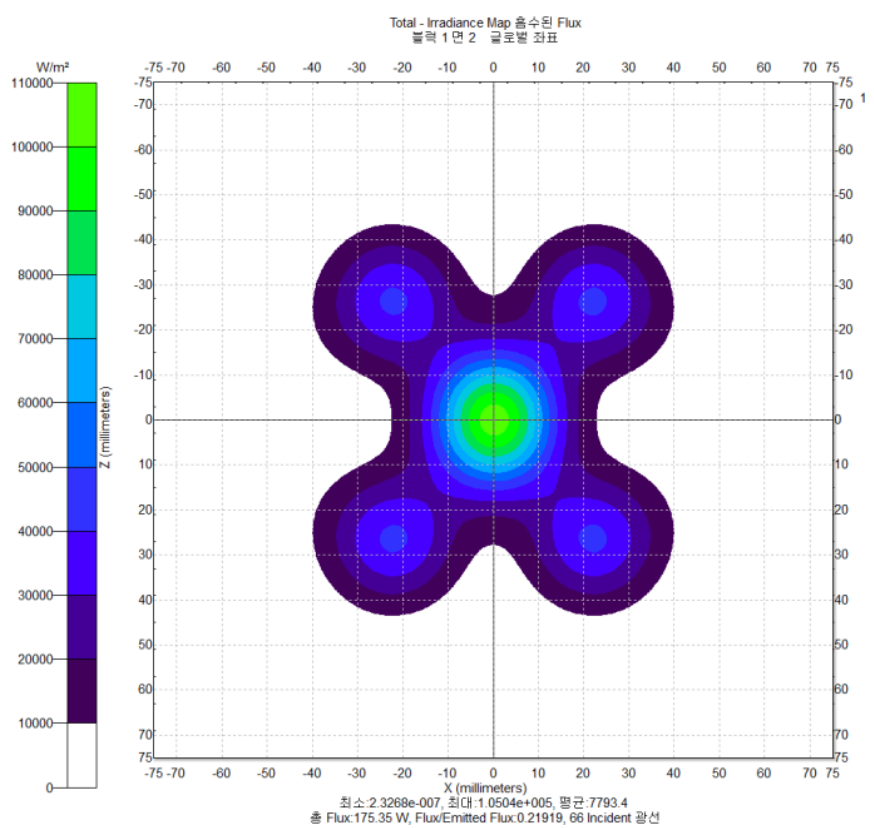

Fig. 13 Distribution of flux with $90 \%$ reflection rate of array-2

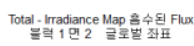
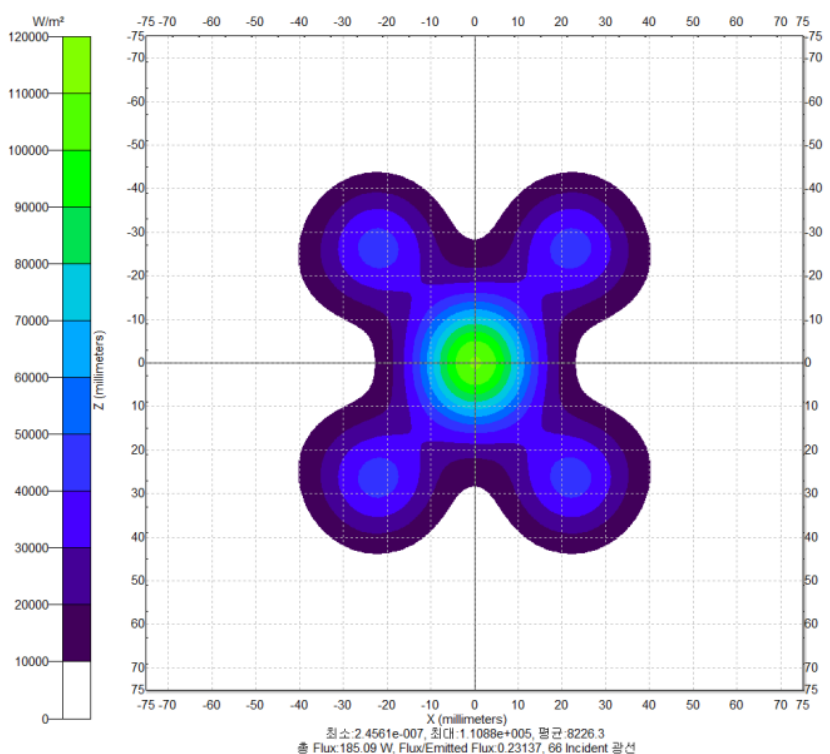

Fig. 14 Distribution of flux with $95 \%$ reflection rate of array-2
Fig. 16-18 show distributions of fluxes reflected by array-3 reflector with 3 different reflection rates. Significant difference between 3 flux distributions cannot be also seen in simulation results. Based on these simulation results, it is possible to know that the reflection rate is not relatively dominant variable. The number of independent reflectors can be a dominant variable in this case.

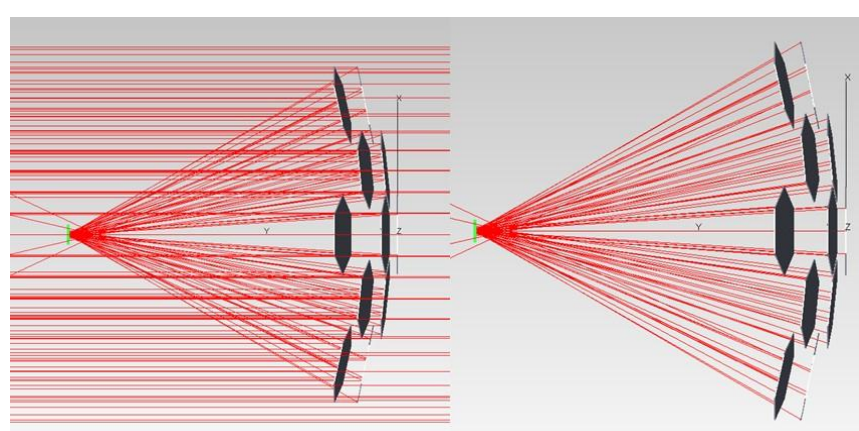

Fig. 15 Simulation of array-3 reflectors

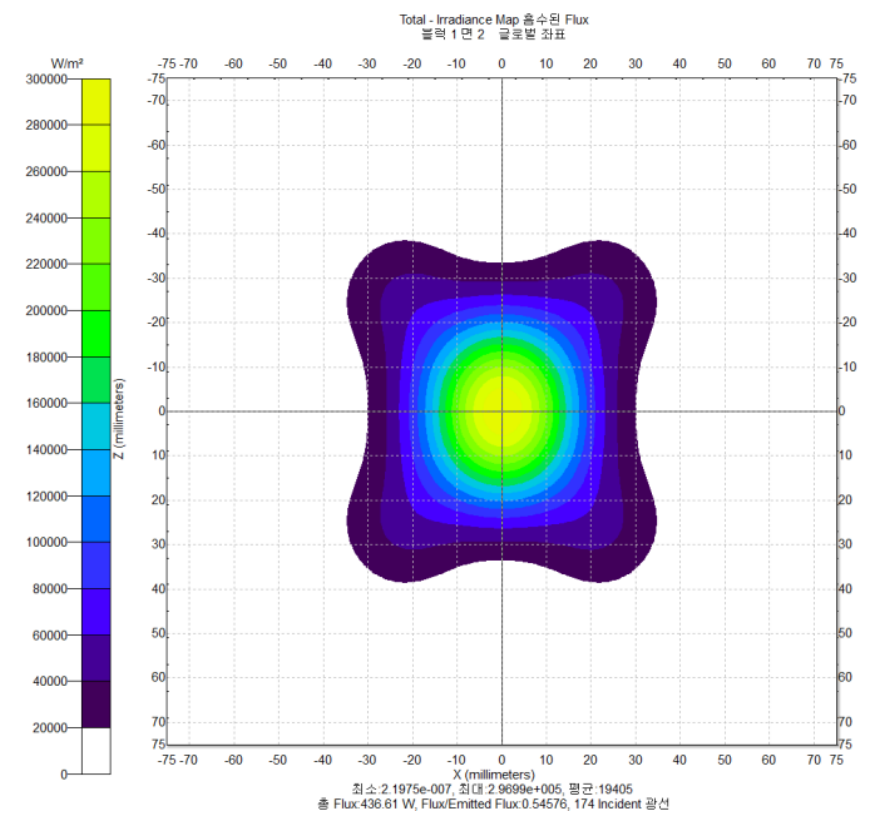

Fig. 16 Distribution of flux with $85 \%$ reflection rate of array-3
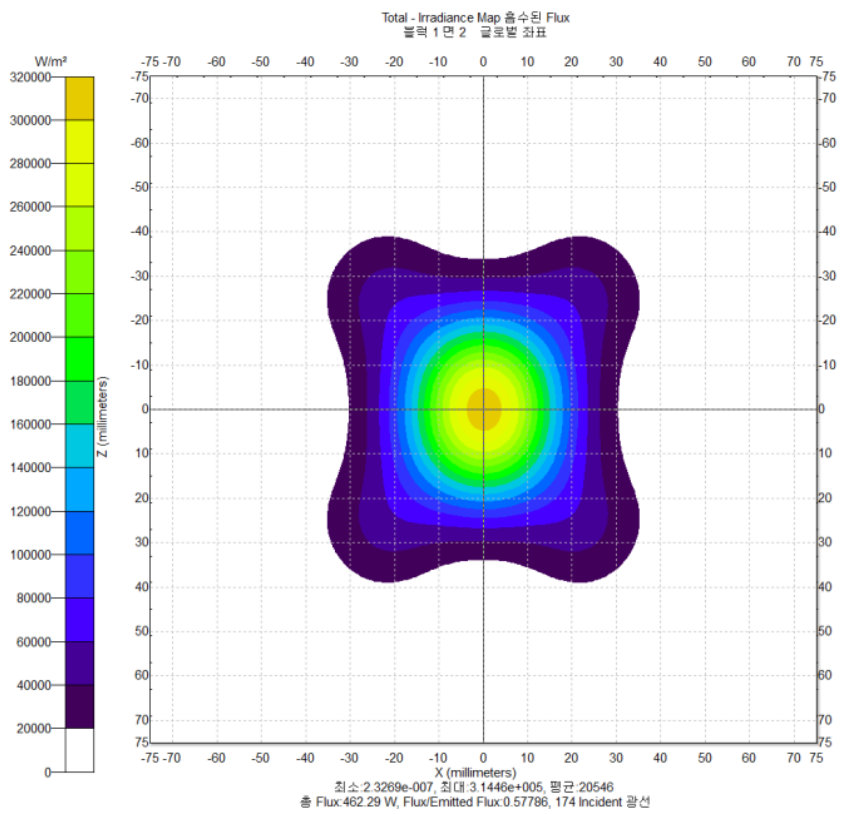

Fig. 17 Distribution of flux with $90 \%$ reflection rate of array-3 


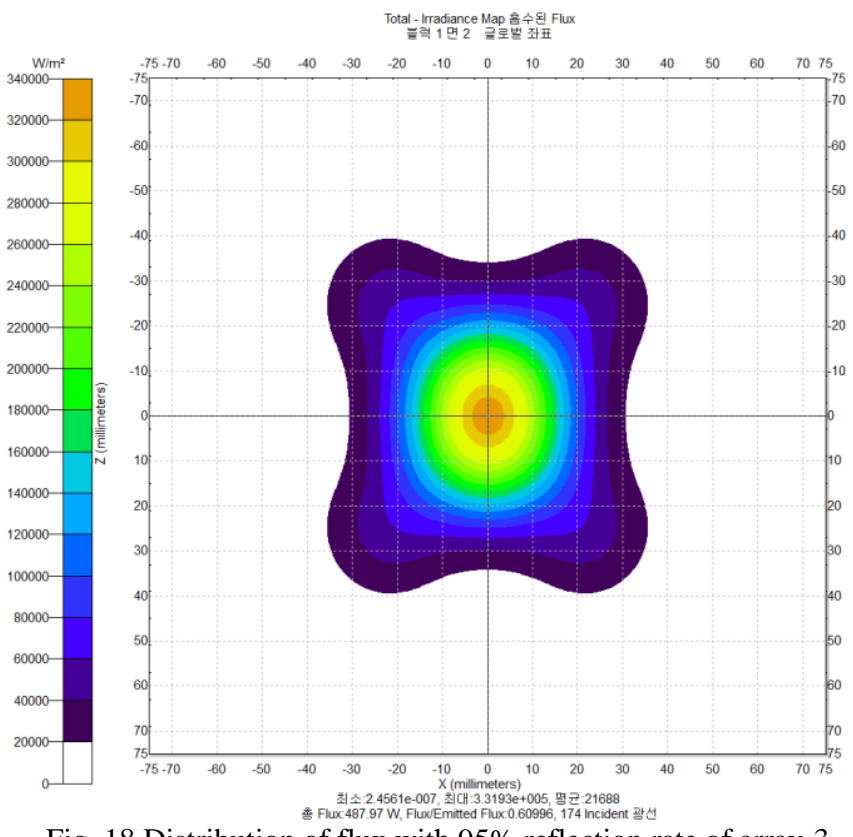

Fig. 18 Distribution of flux with $95 \%$ reflection rate of array-3

Fig. 19 shows fluxes varied with reflection rates and the number of reflectors. The number of reflectors must be considered as dominant variable to effectively collect solar fluxes.

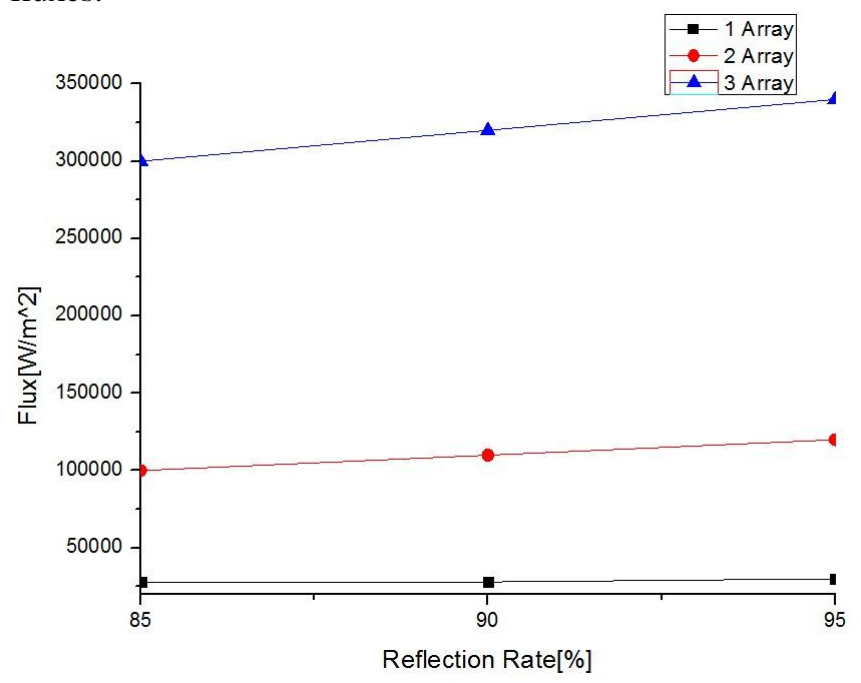

Fig. 19 Fluxes varied with reflection rates and the number of reflectors

\section{CONCLUSION}

In this paper, the objective is determining positions and orientations of all independent reflectors to concentrate all reflected fluxes on a focus point. For this, TracePro was used to analyze behavior of reflected fluxes by the independent reflectors. Therefore conclusion was obtained as follows. Based on these simulation results, it is possible to know that the reflection rate is not relatively dominant variable. The number of independent reflectors can be a dominant variable in this case. The number of reflectors must be considered as dominant variable to effectively collect solar fluxes. Therefore if single module independent reflectors were taken into account in order to develop effective solar collector system, this system must be light-weight because it must effectively support all the reflectors.

\section{ACKNOWLEDGMENT}

This work was supported by the Korea Institute of Energy Technology Evaluation and Planning(KETEP) and the Ministry of Trade, Industry \& Energy(MOTIE) of the Republic of Korea (No. 20143030081160)

\section{REFERENCES}

[1] J. H. Shin, "Performance Characteristics of the Solar Cogeneration System with Multi-Module", Master's degree. Kangwon National University, Republic of Korea, 2014.

[2] D. Mills, "Advances in solar thermal electricity technology", Solar Energy, vol. 76, pp. 19-31, 2004. http://dx.doi.org/10.1016/S0038-092X(03)00102-6

[3] Y. G. Jung, J. G. Lee, J. H. Lee, and Seo, T. B. "Analysis of Heat Transfer Performance for a Dish Type Solar Receiver" The Korean Society of Mechanical Engineers, vol. 2009, pp. 134-137. 2009.

[4] Y. H. Kang, H. Y. Kwak, H. K.Yoon, C. K. Yoo, D. G. Lee, M. C. Kang, and H. S. Yoon. "Flux Distribution of the Dish Concentrator", Journal of the Korean Solar Energy Society, pp. 127-133, 1999.

[5] K. H. Song, C. W. Son, S. H. Lee and T. I. Seo, "Design, Analysis and Empirical Researches for Solar Heat Collecting System based on Flat Mirrors Combination", International Journal of New Technology and Research, vol. 1, pp. 89-92, Oct. 2015.

[6] S. Y. Ryu, "An Analysis of Heat Losses from a Receiver for a Dish-Type Solar Energy Collecting System", Inha University, Korea, 1999.

[7] D. S. Ma, Y. K. Kim and T. B. Seo, "Performance Comparison of Dish Solar Collector with Mirror Arrays \& Receiver Shapes", The Korean Solar Energy Society, pp. 44-49, Nov. 2006. 